\title{
Modified Dual Second-order Generalized Integrator FLL for Frequency Estimation Under Various Grid Abnormalities
}

\author{
Kalpeshkumar R. Patil and Hiren H. Patel
}

\begin{abstract}
Proper synchronization of Distributed Generator with grid and its performance in grid-connected mode relies on fast and precise estimation of phase and amplitude of the fundamental component of grid voltage. However, the accuracy with which the frequency is estimated is dependent on the type of grid voltage abnormalities and structure of the phase-locked loop or frequency locked loop control schemes. Among various control schemes, second-order generalized integrator based frequencylocked loop (SOGI-FLL) is reported to have the most promising performance. It tracks the frequency of grid voltage accurately even when grid voltage is characterized by sag, swell, harmonics, imbalance, frequency variations etc. However, estimated frequency contains low frequency oscillations in case when sensed grid-voltage has a dc offset. This paper presents a modified dual second-order generalized integrator frequency-locked loop (MDSOGI-FLL) for three-phase systems to cope with the nonideal three-phase grid voltages having all type of abnormalities including the de offset. The complexity in control scheme is almost the same as the standard dual SOGI-FLL, but the performance is enhanced. Simulation results show that the proposed MDSOGI-FLL is effective under all abnormal grid voltage conditions. The results are validated experimentally to justify the superior performance of MDSOGI-FLL under adverse conditions.
\end{abstract}

Keywords-Grid synchronization, phase-locked loop, frequency locked loop, second-order generalized integrator.

\section{INTRODUCTION}

Grid synchronization is an adaptive process in which an internal reference signal generated by the synchronization control algorithm allows power converter output signal to work in synchronism with a fundamental component of the grid voltage. Hence, for the converter to perform satisfactorily, it is desired that the control algorithm correctly derives this reference signal from the grid voltages. However, it is a challenging task especially when the grid voltage is characterized by the presence of different grid abnormalities (voltage fluctuation, harmonics, frequency variation and/or distortion, dc offset, switching notches etc.). The issue is more dominant in a microgrid (or a weak grid) where the effect of non-linear loads, faults, sudden load changes etc., are translated in form of distortion in grid voltage, frequency

K. R. Patil is with the Department of Electrical Engineering Sarvajanik College of Engineering \& Technology, Surat, India. (e-mail: kalpesh.patil@scet.ac.in).

H. H. Patel is with the Department of Electrical Engineering, Sarvajanik College of Engineering \& Technology, Surat, India. (e-mail: hiren.patel@scet.ac.in). change, imbalance etc. to a greater extent than that in a stiff or strong grid. Such irregularities may introduce measurement and data conversion errors and hence, makes the task of estimating frequency and synchronization difficult. In addition, the microgrid is likely to have variety of distributed generators (DG) interfaced through static converters, which increases the severity of the problem and makes the task of frequency estimation still more difficult [1-4].

The frequency estimation can be carried out with frequency-domain or time-domain techniques. The frequencydomain techniques based on the discrete Fourier transform (DFT) suffers from the computational burden, accumulation errors etc. Also, complexity in actual implementation increases in case where the measured signal is highly distorted [5-7]. Unlike these methods, several advantages of timedomain techniques like; ease of implementation, reliability, good dynamic response, higher accuracy, low sensitivity to the frequency variations and unbalanced grid voltage etc.; have increased the popularity of the time domain approach based on closed loop phase-locked loop (PLL) for estimating the frequency. The basic structure of a PLL comprises of the phase detector (PD) block, filter and a voltage controlled oscillator (VCO). The PD is responsible for adjusting the gain of VCO whose output signal is synchronized (in terms of phase and frequency) with the input reference signal. The classification of PLL with different PD is discussed in [8-11]. Their performances in single-phase power system network are evaluated under the different grid severity. The three-phase conventional PLL based on the synchronous reference frame (SRF) have superior performance in the balanced grid voltage signal. But, it gives the oscillatory response for the estimated fundamental frequency, if measured grid voltage signal is unbalanced or distorted [12]. The poor performance of the conventional PLL was improved by the technique reported in [13], which employs decoupled double synchronous reference frame PLL (DDSRF-PLL). The DDSRF-PLL decouples the fundamental positive and negative sequence components to estimate the frequency. It results into accurate estimation in case of unbalanced grid voltage signal. However, performance deteriorates when grid signal is distorted. It is observed that PD structure based on the generalized integrator (GI) have a better performance and reliability [14] under such distorted gird signal conditions. Hence, the second-order generalized integrator based PLL (SOGI-PLL) is superior to other configurations. It performs fast and accurately under the adverse grid conditions. Unlike SOGI-PLL where the 
estimated frequency is used as feedback from the PLL block in the structure of SOGI block, the frequency can be adaptively obtained through frequency-locked loop (FLL) in SOGI-FLL structure to get superior performance. This avoids the PLL and the adaptive nature enhances the performance [15-18]. Two SOGI blocks connected in parallel forming a dual SOGI-FLL (DSOGI-FLL) structure provides good transient response even in the presence of grid abnormalities (i.e. frequency variation, unbalanced voltages etc.). However, performance with distorted grid signals i.e. when harmonics are present is not satisfactory.

Apart from these disturbances, presence of dc component may also affect the performance of synchronization schemes. The probable source of dc component can be the measurement errors, conversion errors resulting through the data converters, inaccurate signal conditioning circuits, injection from DG due to their improper control, saturation of magnetic components etc. The performance of conventional PLLs, DSOGI-PLL, DSOGI-FLL is severely hampered in presence of the dc offset. The estimated frequency under such case is characterized by presence of low frequency component superimposed on the average value.

This aspect (frequency estimation in presence of the dcoffset) is not fully explored. To target this issue, few methods have been reported [19-24]. However, they are complex and suggested for single-phase system. Hence, in order to eliminate errors in frequency estimation caused due to presence of the dc offset and other grid abnormalities (e.g. unbalance, harmonics, frequency variation, magnitude variation etc.), a modified DSOGI-FLL (MDSOGI-FLL) for three-phase systems is proposed [25]. The modified SOGIQSG structure used in the proposed MDSOGI-FLL employs the third integrator to estimate dc component and overcomes its effect in the estimation of frequency. The performance analysis of this structure has been carried out under presence of different grid voltage abnormalities and the superior performance is illustrated through simulation and experimental results. The details of the MDSOGI-FLL and its capability to perform under different conditions are highlighted in the remaining sections.

\section{MODIFIED DSOGI-FLL}

The section presents the basic configuration and reports the transfer functions of DSOGI-FLL [8] and proposed MDSOGIFLL. In addition, the procedure for tuning of gain for both these FLL for estimating frequency under various grid abnormal conditions is also presented.

\section{A. DSOGI-FLL: Structure and Transfer Functions}

Fig. 1(a) shows structure of conventional DSOGI-FLL where the three-phase utility voltages $\left(V_{a s}, V_{b s}\right.$, and $\left.V_{c s}\right)$ under balanced condition are defined as

$$
V_{a b c s}=V_{m} \times\left(\begin{array}{c}
\sin \omega t+\emptyset_{a} \\
\sin \left(\omega t-\frac{2 \pi}{3}\right)+\emptyset_{b} \\
\sin \left(\omega t+\frac{2 \pi}{3}\right)+\emptyset_{c}
\end{array}\right)
$$

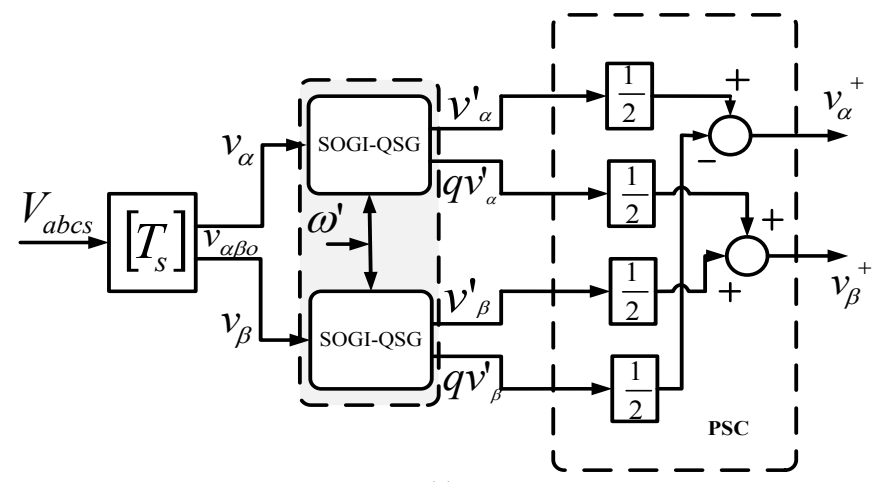

(a)

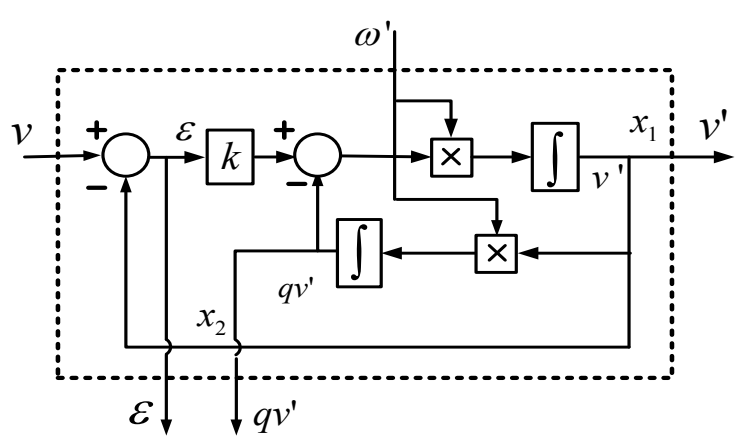

(b)

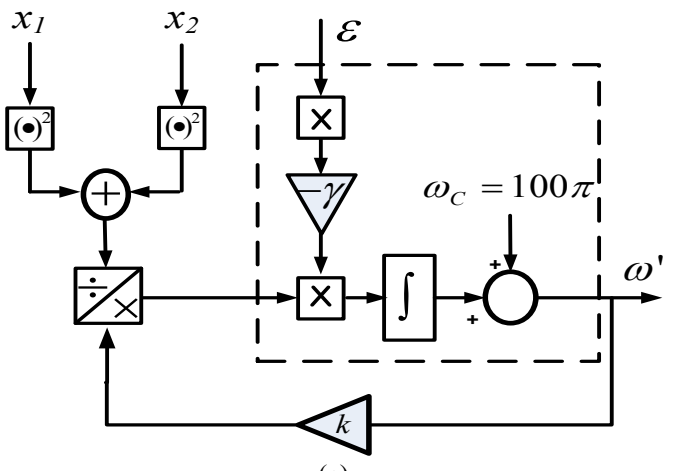

(c)

Fig. 1. Basic structure of DSOGI-FLL: (a) abc to $\alpha^{+} \beta^{+}$computation(b) Standard SOGI-QSGs and (c) FLL-block.

$V_{a b c s}=\left[\mathrm{V}_{\mathrm{as}} \mathrm{V}_{\mathrm{bs}} \mathrm{V}_{\mathrm{cs}}\right]^{\mathrm{T}}$ and $\mathrm{V}_{\mathrm{m}}, \omega$ and $\varnothing$ are the amplitude, angular frequency and phase difference of the grid signal voltage, respectively. Under the assumption of the balanced utility voltages (1) can be represented in stationary reference frame by (2) as

$$
v_{\alpha \beta 0}=\left[T_{s}\right] \times V_{a b c s}
$$

where $v_{\alpha \beta}=\left[v_{\alpha}, v_{\beta}\right]^{\mathrm{T}}$ and $\left[T_{s}\right]$ denotes the Clarke transformation matrix represented by (3)

$$
\left[T_{s}\right]=\frac{2}{3}\left[\begin{array}{ccc}
1 & -\frac{1}{2} & -\frac{1}{2} \\
0 & \frac{\sqrt{3}}{2} & -\frac{\sqrt{3}}{2} \\
\frac{1}{\sqrt{2}} & \frac{1}{\sqrt{2}} & \frac{1}{\sqrt{2}}
\end{array}\right]
$$


The Clarke or $\alpha \beta 0$ transform is a space vector transformation of time-domain signals from a natural three-phase coordinate system $(a b c)$ into a two-phase stationary reference frame $\alpha \beta 0$. The proposed MDSOGI-FLL requires two in-quadrature components in a stationary reference frame, which are thus derived using Clarke transformation.

The shaded block showing two SOGI-QSG that represents dual second-order generalized integrators is the key part of PLL or FLL structure. The details of SOGI-QSG are shown in Fig. 1(b). The $v^{\prime}$ and $q v^{\prime}$ signals forms a pair of quadrature signals where $q v^{\prime}$ lags $v^{\prime}$ by $90^{\circ}$. These signals are used to extract positive sequence components through the Positive Sequence Calculator (PSC shown in Fig. 1(a)) in the $\alpha \beta$ stationary reference frame. The positive sequence components $v_{\alpha}^{+}$and $v_{\beta}^{+}$can be utilized to estimate the positive sequence $\left(v_{a b c s}^{+}\right)$of the distorted grid input signal. Apart from filtering, SOGI-QSGs also work as voltage controlled oscillator [10] and thus the approach avoids extra voltage controlled oscillator unlike that in SRF based PLL. A simple auto tune block shown in Fig. 1(c) is used to adapt the center frequency $\omega$ ' of the SOGI resonator to the input frequency. The frequency is estimated through the Frequency-Locked Loop (FLL) block adaptively by adjusting the gain $(\gamma)$ in DSOGIFLL and thus it discards the PLL block used in DSOGI-PLL. Hence, in the grid abnormalities like sag, swell, variations, phase jump, harmonics etc., the DSOGI-FLL performs extremely well, fast and precisely as compared to conventional SRF-PLL based schemes.

The transfer functions represented by (4) and (5) for bandpass filter (BPF) and low-pass filter (LPF), respectively, are obtained from the standard SOGI-QSGs shown in Fig.1 (b). They characterize the adaptive filtering structure of it. The bandwidth (or sharpness) of the band-pass filter $D(s)$ and low-pass filter $Q(s)$, can be adjusted by proper tuning of the real positive value of gain $k$. Hence, the signals $v^{\prime}$ and $q v^{\prime}$ are the outputs of the BPF and LPF, respectively, with $90^{\circ}$ phase shift between them. Actually, the LPF has a better filtering feature to the high frequencies than the BPF. But the $q v^{\prime}$ output of this standard SOGI-QSGs suffers from nonzero dc offset present in input grid voltage or the measured grid voltage. It is reflected in the calculation of positive sequence $v_{\beta}^{+}$as shown in Fig. 2, which shows the response of $v_{\alpha}^{+}$and $v_{\beta}^{+}$when sudden frequency change and dc offset are

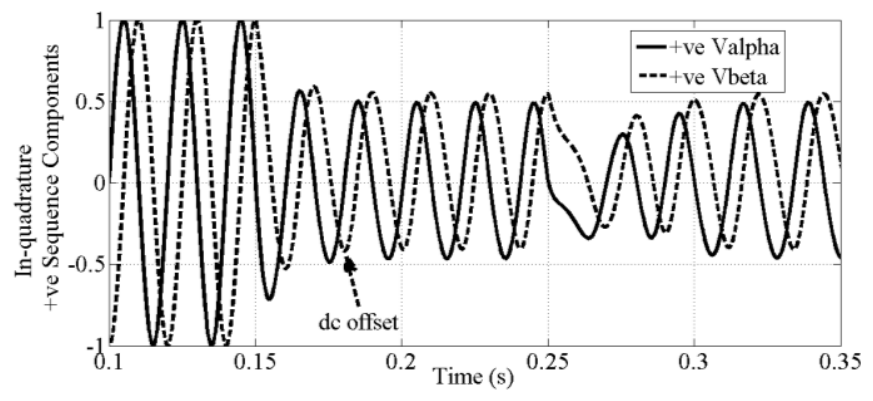

Fig. 2. Output waveform of in-quadrature $\left(v_{\alpha}^{+}\right.$and $\left.v_{\beta}^{+}\right)$positive sequence components in presence of dc offset and frequency jump in the sensed input signal of standard SOGI-QSGs. introduced. This leads to inaccurate estimation of fundamental positive sequence components and frequency.

$$
\begin{aligned}
& D(s)=\frac{v^{\prime}}{v}(s)=\frac{k \omega^{\prime} s}{s^{2}+k \omega^{\prime} s+\omega^{\prime 2}} \\
& Q(s)=\frac{q v^{\prime}}{v}(s)=\frac{k \omega^{\prime 2}}{s^{2}+k \omega^{\prime} s+\omega^{\prime 2}}
\end{aligned}
$$

\section{B. MDSOGI-FLL: Structure and Transfer Functions}

It is worth noting from the above section that the conventional DSOGI-FLL fails to estimate the frequency accurately when sensed grid signals are characterized by the dc offset. The proposed MDSOGI-FLL structure is similar to that of DSOGI-FLL shown in Fig.1 (a) except the fact that the

SOGI-QSGs (Fig. 1(b)) are now replaced by the modified SOGI-QSGs shown in Fig. 3.

The modified SOGI-QSGs of Fig. 3 can be described by the following transfer functions (6), (7) and (8).

$$
\begin{gathered}
D^{\prime}(s)=\frac{v^{\prime}(s)}{v(s)}=\frac{k_{1} \omega^{\prime} s^{2}}{\Delta(s)} \\
Q^{\prime}(s)=\frac{q v^{\prime}(s)}{v(s)}=\frac{k_{1} \omega^{\prime 2} s}{\Delta(s)} \\
V_{d c}(s)=\frac{v_{d c}(s)}{v(s)}=\frac{k_{d c} \omega^{\prime}\left(s^{2}+\omega^{\prime 2}\right)}{\Delta(s)}
\end{gathered}
$$

where

$$
\Delta(s)=s^{3}+\left(k_{1}+k_{d c}\right) \omega^{\prime} s^{2}+\omega^{\prime 2} s+k_{d c} \omega^{3}
$$

It is depicted from (6) and (7) that $D^{\prime}(s)$ and $Q^{\prime}(s)$ both have a band pass filtering characteristic and results into the rejection of offset in the in-quadrature components. However, proper tuning of gain parameters $k_{1}$ and $k_{d c}$ is necessary for it.

\section{Gain Tuning of SOGI-QSG and Proposed Structure}

The sub-section presents the analysis for determination of the parameters involved in the LPF and BPF transfer functions of DSOGI-FLL and MDSOGI-FLL.

\section{i. Tuning of Standard SOGI-QSG}

From (4), the characteristic equation $s^{2}+k \omega^{\prime} s+\omega^{\prime 2}=0$ is

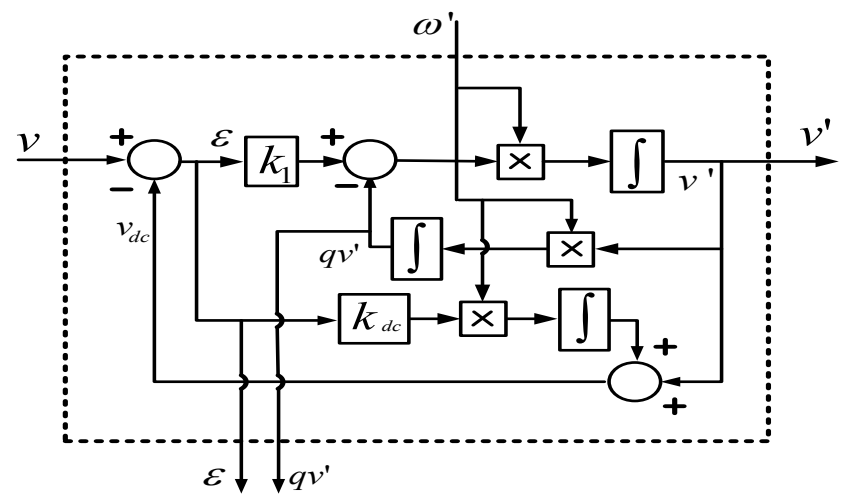

Fig. 3 Block diagram of Modified SOGI-QSG block 


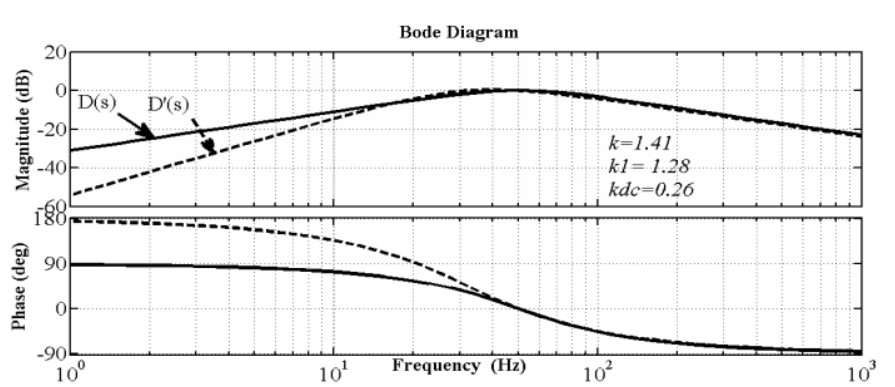

(a)
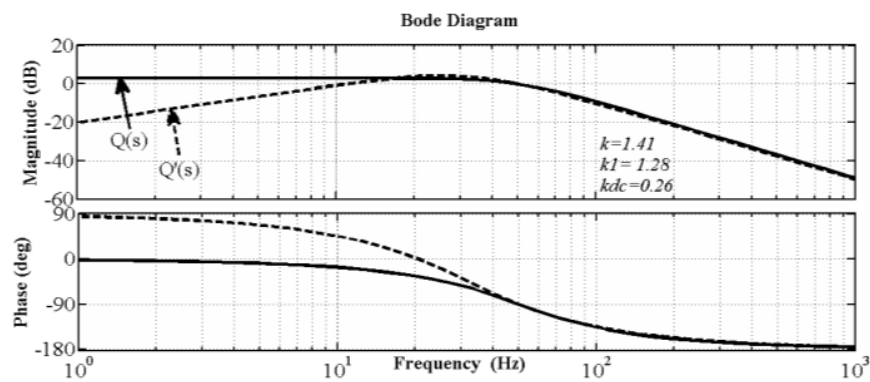

(b)

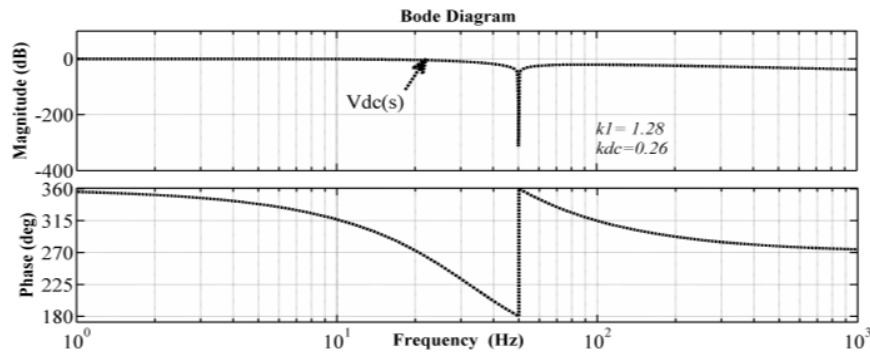

(c)

Fig. 4. Comparison of the frequency response for the transfer function (a) $D(s)$ and $D^{\prime}(s)$ (b) $Q(s)$ and $Q^{\prime}(s)$ (c) $V_{d c}(s)$.

compared with the standard characteristic equation $s^{2}+$ $2 \zeta \omega_{n} s+\omega_{n}^{2}=0$ to obtain the gain $k$ at the damping ratio $\zeta=0.707$ (underdamped system) and natural frequency of oscillation $\omega_{n}=2 \pi 50 \mathrm{rad} / \mathrm{s}$. The gain $k$ is given by (10).

$$
k=\frac{9.2}{t_{s} \omega^{\prime}}
$$

where settling timet $t_{s}=4.6 \times \tau ; \tau=\frac{1}{\zeta \omega_{n}}$

It is essential to have a proper tuning of the gain $k$ to have appropriate bandwidth to discard the low frequency and higher frequency components present in the input signals. Figs. 4(a) (b) and (c) show bode plots for $D(s), Q(s)$ and $V_{d c}(s)$ (represented by (8)), obtained using (10)-(11), for $k=1.41$ and $\zeta=0.707$. It is evident from these plots that $D(s)$ attenuates the low frequency components unlike $Q(s)$ and hence, the dc offset is not eliminated $\operatorname{in} v_{\beta}^{+}$.

\section{ii. Tuning of Proposed Modified SOGI-QSG}

The parameters $k_{l}$ and $k_{d c}$ shown in Fig.3 are selected based on the roots of the $\Delta(s)$ assuming that all the roots have equal real parts (all three poles have equal natural frequency of oscillation). These parameters are obtained by comparing the $\Delta(s)$ represented by (9) with (12).

$$
\left(s+\omega_{n_{1}}\right)\left(s^{2}+2 \zeta \omega_{n_{2}} s+\omega_{n_{2}}^{2}\right)=0
$$

To estimate the gain $k_{1}$ and $k_{d c}, \omega_{n_{1}}=\omega_{n_{2}}=2 \pi 50 \mathrm{rad} / \mathrm{s}$ and $\zeta=0.707$ (same as that considered for tuning DSOGI- FLL) is considered. The effect of both the gain adjustment is clearly indicated in the bode diagrams of Fig. 4. It is observed from Figs. 4(a) and (b) that for low frequencies, magnitude bode plots of $D^{\prime}(s)$ and $Q^{\prime}(s)$ lie below $0 \mathrm{~dB}$, indicating attenuation of low frequency components and dc component. The magnitude plots of $D(s)$ and $Q(s)$ are also shown in Figs. 4(a) and (b) to compare the performance of DSOGI-FLL and MDSOGI-FLL. It is evident that as gain $Q(s)$ is positive, it does not attenuate dc component. Fig. 4(c) shows bode plot for (8) that represents the performance of third generalized integrator. The magnitude of transfer function $V_{d c}(\mathrm{~s})$ is nearly $0 \mathrm{~dB}$ till $50 \mathrm{~Hz}$ indicating no effect on the computation of the low frequency components. However, sudden decrease in the magnitude (large negative magnitude) is observed at $50 \mathrm{~Hz}$, which indicates the attenuation of $50 \mathrm{~Hz}$ (fundamental) component. The attenuation of higher order harmonics also occurs as negative gain is observed for $V_{d c}(S)$ at frequencies higher than $50 \mathrm{~Hz}$. However, it is much lower than that at $50 \mathrm{~Hz}$. Thus, the inclusion of third integrator results into large attenuation of fundamental component (also higher order frequencies to a certain extent) allowing only the low order frequencies to pass. Thus, it helps to estimate and eliminate the dc offset accurately from input $v$ of Fig. 3.

\section{Simulation ReSUlts}

The effectiveness of the proposed MDSOGI-FLL is demonstrated through the simulation results obtained in MATLAB/Simulink. The parameter values used in the modified DSOGI-FLL simulation model are as follows: $k_{l}=1.28, k_{d c}=0.26$ and $\gamma=40$. The performance is evaluated for three cases: (i) balanced sag, (ii) imbalance in three-phase supply voltage, and (iii) presence of harmonics. In all the cases it is considered that the dc offset is present due to the measurement or data conversion errors.

Fig. 5(a) presents the case (i) where a step change in amplitude of the grid signal is observed at $\mathrm{t}=0.15 \mathrm{~s}$, where the amplitude decreases from 1per unit (pu) to $0.5 \mathrm{pu}$. Also the dc offset of $0.1 \mathrm{pu}$ is introduced at $\mathrm{t}=0.15 \mathrm{~s}$. In addition, at $\mathrm{t}=0.25 \mathrm{~s}$ frequency of grid signal suddenly changes from $50 \mathrm{~Hz}$ $(314 \mathrm{rad} / \mathrm{s})$ to $45 \mathrm{~Hz}(282.6 \mathrm{rad} / \mathrm{s})$.

Fig. 5(b) shows that both $v_{\alpha}^{+}$and $v_{\beta}^{+}$for MDSOGI-FLL are free from offset, unlike that observed for DSOGI-FLL in (Fig. 2 ). The reason being the band-pass filtering capabilities of $D^{\prime}(s)$ and $Q^{\prime}(s)$, unlike DSOGI-FLL where $D(s)$ behaves as BPF while $Q(s)$ behaves as LPF. It is observed from Fig. 5(c), that the DSOGI-FLL is able to track the frequency accurately only till $\mathrm{t}=0.15 \mathrm{~s}$, and then exhibits oscillatory nature. Also the large dip in the estimated frequency is observed at $\mathrm{t}=0.25 \mathrm{~s}$. Unlike the DSOGI-FLL, the MDSOGI-FLL does not show oscillations and estimated frequency quickly settles down to the final value. The dips at the instant of step changes in 


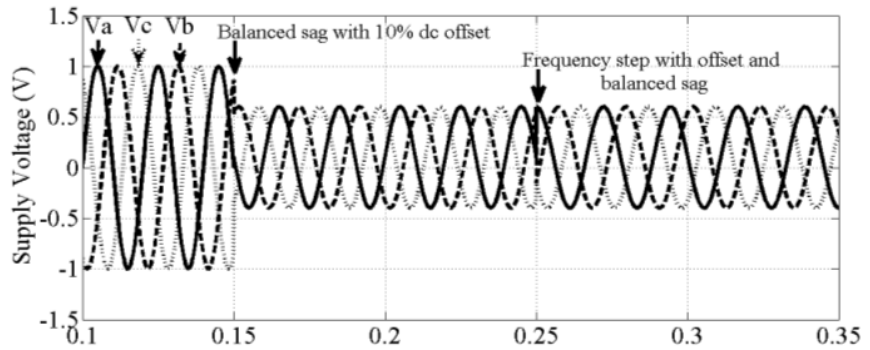

(a)

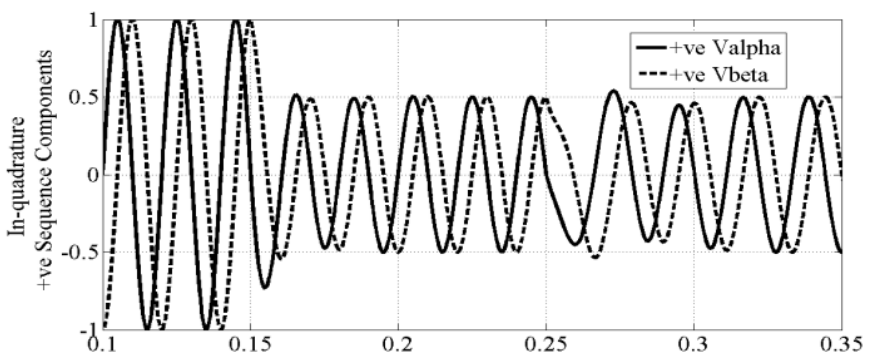

(b)

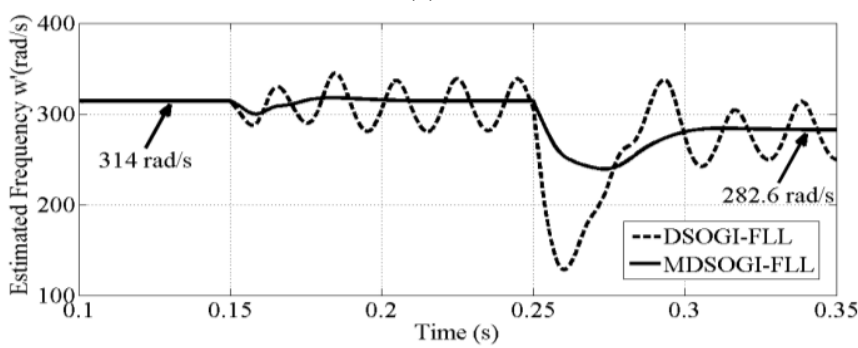

(c)

Fig. 5. Performance under balanced sag conditions: (a) Three-phase grid voltage signals (b)in-quadrature $\left(v_{\alpha}^{+}\right.$and $\left.v_{\beta}^{+}\right)$positive sequence components (c) Estimated frequency.

amplitude of grid voltage and frequency change are also much less than that observed with MDSOGI-FLL.

Unlike case (i), for the case (ii) shown in Fig. 6, step change in amplitude of the grid signal is considered only for phase ' $a$ '. At $\mathrm{t}=0.15 \mathrm{~s}$, the amplitude of phase ' $\mathrm{a}$ ' voltage decreases from $1 \mathrm{pu}$ to $0.5 \mathrm{pu}$. All other changes are similar to that in case (i).The ripple in the estimated frequency of DSOGI-FLL is less than the earlier case. However, the nature is similar to that observed in case (i). The MDSOGI-FLL once again shows superior performance. Fig. 7(a) shows the grid voltage characterized by the presence of $5^{\text {th }}, 7^{\text {th }}, 11^{\text {th }}$, and $13^{\text {th }}$ harmonics alongwith the dc offset of $0.1 \mathrm{pu}$ after $\mathrm{t}=0.15 \mathrm{~s}$. The step change in frequency is applied at $\mathrm{t}=0.25 \mathrm{~s}$ as in case (i)and case (ii). Fig. 7(b) displays oscillations in the estimated frequency of DSOGI-FLL, while the proposed MDSOGI-FLL eliminates the oscillation caused by the dc offset. As a result, the grid signal frequency is quickly and accurately tracked.

Fig. 8 shows the comparison of different frequency estimation techniques when subjected to a step change in the supply frequency. The supply voltage is considered to have the harmonics, unbalance and the dc-offset. The comparison is carried out for $\omega_{\mathrm{n}}=2 \pi 50 \mathrm{rad} / \mathrm{s}, \zeta=0.707$ to have the settling time of $40 \mathrm{~ms}$. Table I shows the settling time for these frequency estimation techniques in -PLL are relatively faster than other techniques. It must response to a step change in the frequency at $\mathrm{t}=0.25 \mathrm{~s}$. SRF-PLL and MDSOGI be noted that

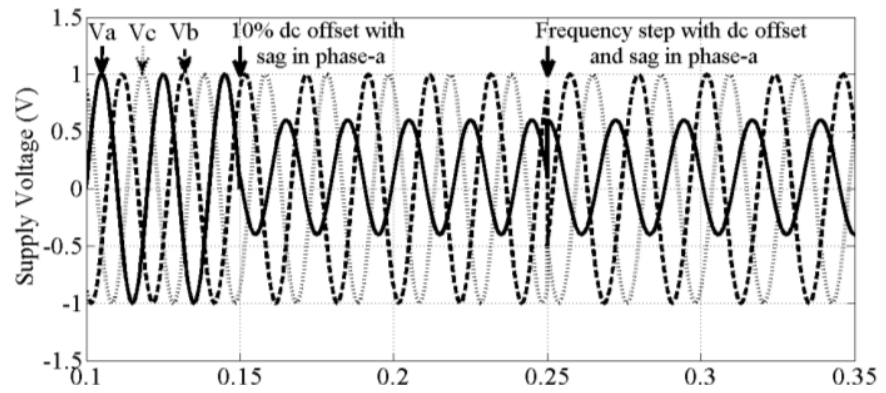

(a)

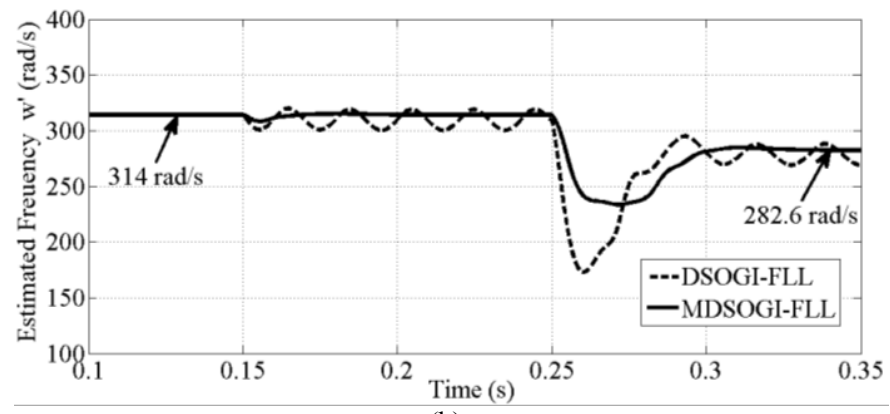

(b)

Fig. 6. Performance under unbalanced sag conditions: (a) Three-phase grid voltage signals (b) Estimated frequency.

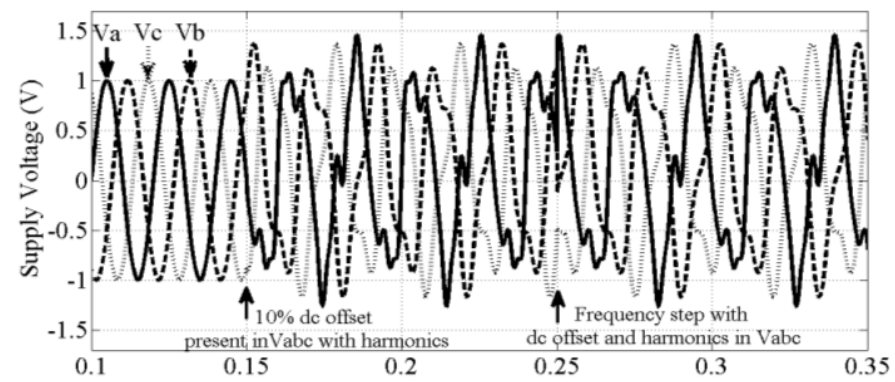

(a)

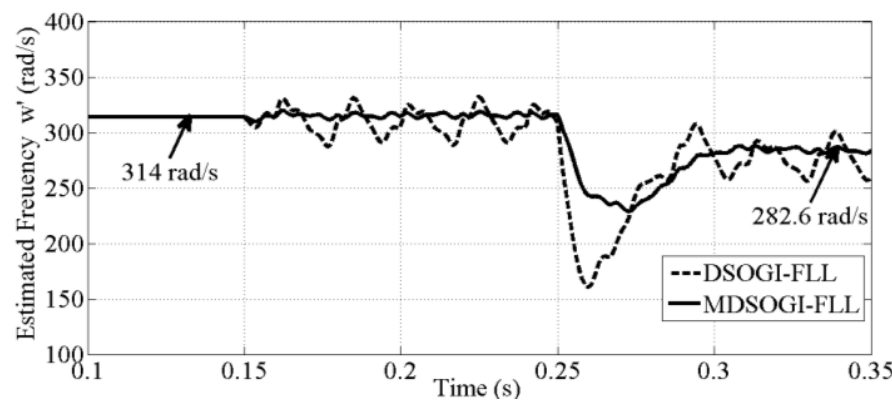

(b)

Fig. 7. Performance when harmonics are present: (a) Three-phase grid voltage signals (b) Estimated frequency with DSOGI-FLL and MDSOGIFLL.

except MDSOGI-FLL all other techniques show sustained oscillations in the estimated frequency. Hence, the settling time for these techniques is determined as the time from the step change till the estimated frequency waveform reaches a stage where sustained oscillations with a constant average (dc) value is achieved. The qualitative comparison of these frequency estimation techniques is also provided in Table II to show their capabilities of detecting and rejecting the effect of harmonics, unbalance and dc-offset in the frequency estimation. The proposed MDSOGI-PLL performs well on all 
the aspect even when the dc-offset is present. Thus, it is not only fast but also accurate under all grid abnormalities.

TABLE I

TIME RESPONSE FOR DIFFERENT FREQUENCY ESTIMATION TECHNIQUES

\begin{tabular}{|c|c|c|}
\hline PLL-Type & Estimated Time (ms) & $\begin{array}{c}\text { Observed Time } \\
(\mathrm{ms})\end{array}$ \\
\hline SRF-PLL & 40 & 50 \\
\hline DDSRF-PLL & 40 & 70 \\
\hline DSOGI-PLL & 40 & 45 \\
\hline DSOGI-FLL & 40 & 60 \\
\hline MDSOGI-FLL & 40 & 50 \\
\hline
\end{tabular}

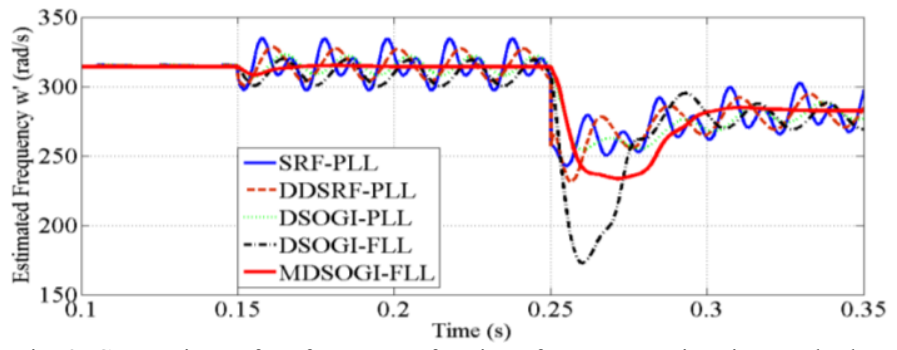

Fig. 8. Comparison of performance of various frequency estimation methods.

TABLE II

PERformance EVAluation Of DifFERENT PLl TeChNiques

\begin{tabular}{|c|c|c|c|}
\hline PLL-Type & $\begin{array}{l}\text { Detection of Negative } \\
\text { Sequence Components }\end{array}$ & $\begin{array}{c}\text { Harmonic } \\
\text { Detection }\end{array}$ & $\begin{array}{c}\text { DC offset } \\
\text { Rejection }\end{array}$ \\
\hline SRF-PLL & No & No & No \\
\hline DDSRF-PLL & Yes & $\begin{array}{c}\text { Requires more } \\
\text { decoupling term }\end{array}$ & No \\
\hline DSOGI-PLL & Yes & Yes & No \\
\hline DSOGI-FLL & Yes & Yes & No \\
\hline MDSOGI-FLL & Yes & Yes & Yes \\
\hline
\end{tabular}

\section{EXPERIMENTAL RESULTS}

The experimental results are included in this section to validate the effectiveness of the proposed MDSOGI-FLL. The cases considered for simulation are verified experimentally using DSpace DS1104 DSP board as control system. The values of various parameters for which the MDSOGI-FLL is tuned are $k_{l}=1.28 k_{d c}=0.26$ and $\gamma=40$, while those for DSOGI-FLL are $k=1.41$ and $\gamma=100$. The fundamental frequency of the three phase supply voltage and the sampling frequency are $50 \mathrm{~Hz}$ and $10 \mathrm{kHz}$, respectively. Thus, the same parameters that are considered for simulation through MATLAB/Simulink are adopted for experimental verification.

Fig.9 shows the three-phase supply voltage along with the frequency estimated by MDSOGI-FLL. The supply, shown in Fig. 9(a) is initially balanced with $50 \mathrm{~Hz}$ and then undergoes the disturbances similar to that shown in Fig. 5(a). Thus, the balanced sag of $50 \%$ and even a frequency step change of $10 \%$ (frequency change from $50 \mathrm{~Hz}$ to $45 \mathrm{~Hz}$ ) is applied in the supply voltage. The dc-offset of $10 \%$ is superimposed on the supply voltage. The supply frequency estimated by both proposed MDSOGI-FLL and the conventional DSOGI-FLL are shown in Fig.9 (b). Just like simulation results, the experimental results also depict oscillations in the frequency estimated by the DSOGI-FLL, while MDSOGI-FLL is free from oscillations and is able to correctly estimate the frequency. The difference ' $\Delta \mathrm{V}$ ', marked by the difference in the position of the two cursors in Fig.9(b), indicates the frequency change of $32 \mathrm{rad} / \mathrm{s}$ corresponding to change of $5 \mathrm{~Hz}$
$(2 \times 3.14 \times 5=31.4 \mathrm{rad} / \mathrm{s})$ when a step change of frequency from $50 \mathrm{~Hz}$ to $45 \mathrm{~Hz}$ (and vice-versa) is applied. The minor variation in the magnitude is due to the limitation of the oscilloscope's resolution. It is clearly evident even from the experimental results that the effect of offset present in supply voltage is very effectively eliminated in the proposed scheme.

Fig. 10 shows the comparison of the proposed MDSOGIFLL structure against the conventional DSOGI-FLL under the conditions similar to that shown in Fig. 6(a). Voltage sag of $50 \%$ is introduced in just one of the phases while the dc offset is present in all the three phases. The three-phase unbalanced voltage signals with above characteristics, used to study the performance of DSOGI-FLL and MDSOGI-FLL, are shown in Fig. 10(a). Fig. 10(b) represents the comparison of the estimated frequency by both these methods. Frequency obtained through DSOGI-FLL approach once again shows that

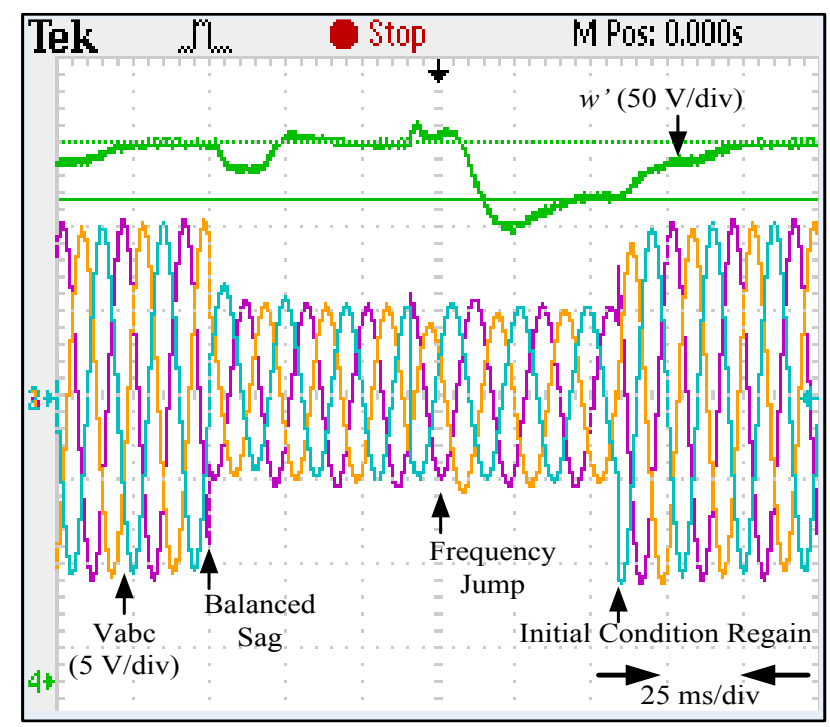

(a)

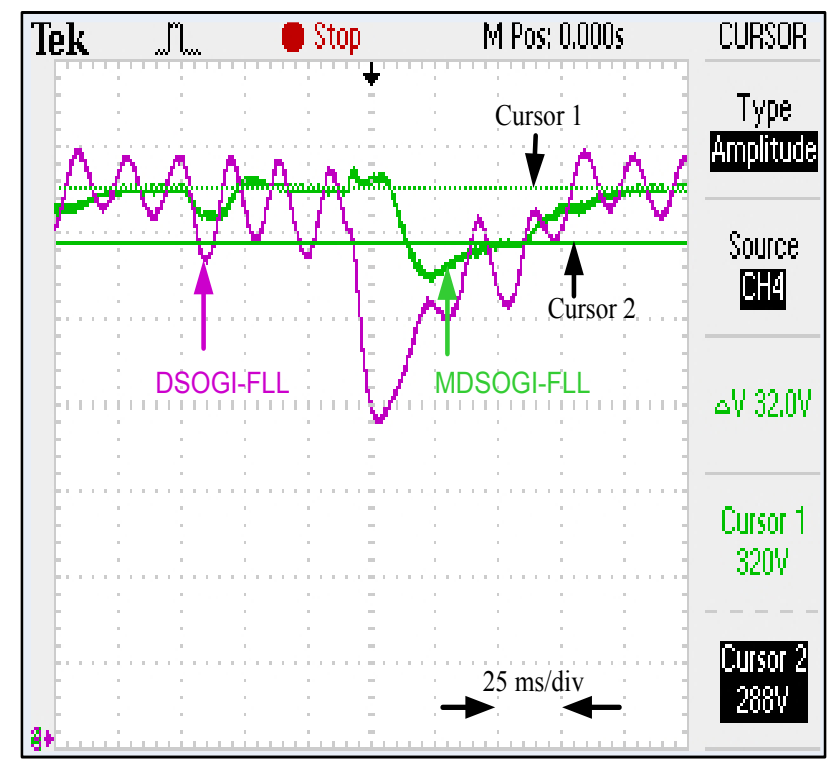

(b)

Fig. 9. Experimental results with balanced-sag and dc-offset in supply voltage: (a) Three-phase supply voltage signals (b) Estimated frequency with DSOGI-FLL and MDSOGI-FLL. 


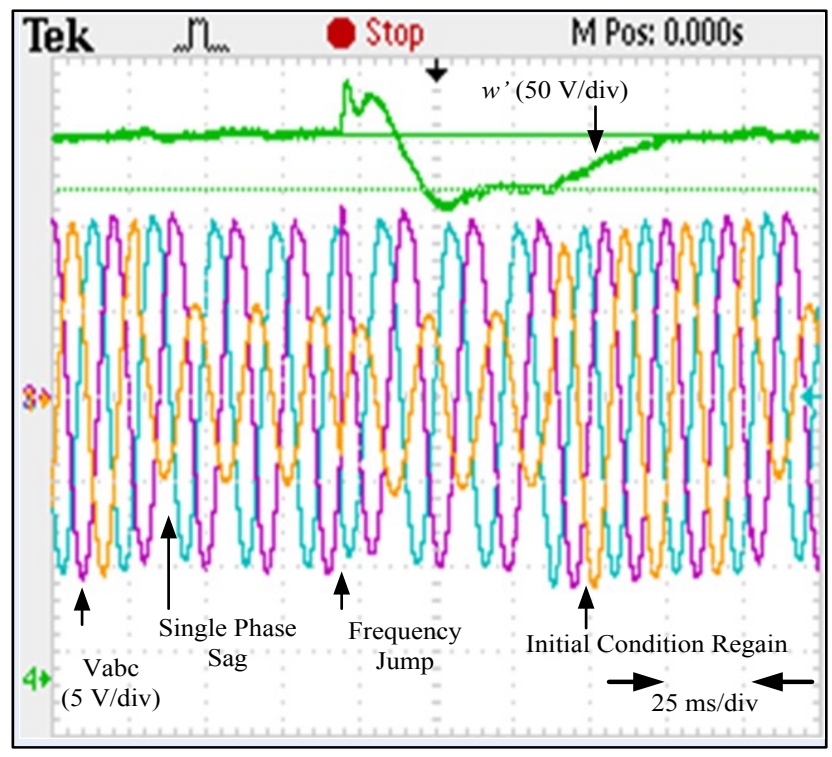

(a)

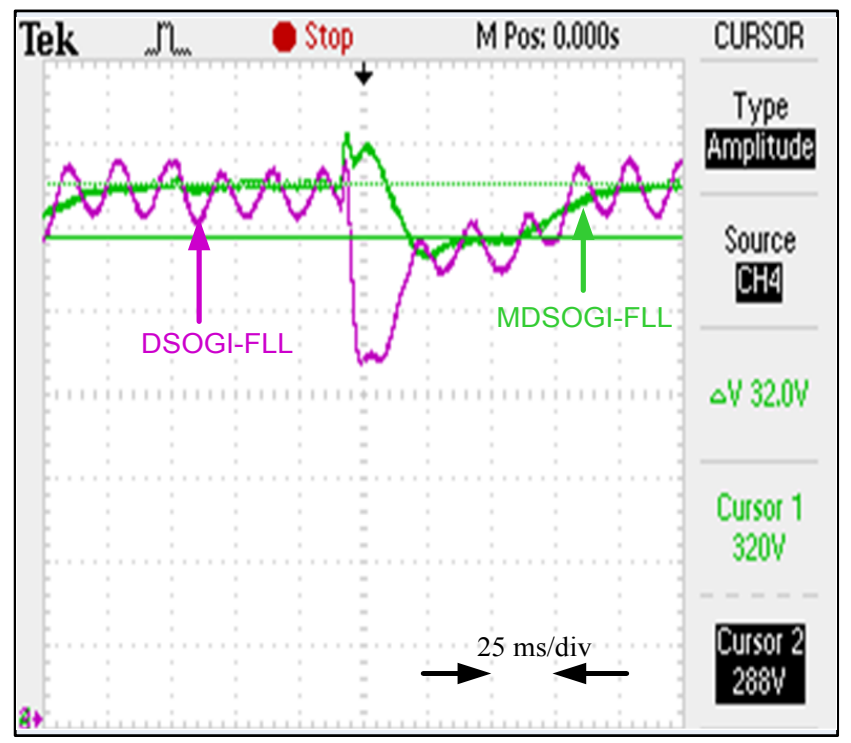

(b)

Fig. 10. Experimental results with imbalance and dc-offset in supply voltage: (a) Three-phase grid voltage (b) Estimated frequency with DSOGI-FLL and MDSOGI-FLL.

the estimated frequency is characterized by an oscillatory response, with a $100 \mathrm{~Hz}$ frequency ripple. Frequency estimated by MDSOGI-FLL not only is free from oscillations, but also has lower frequency dip corresponding to sudden frequency decrease. Thus, the overshoot with the step response with MDSOGI-FLL is less indicating a better transient response.

The immunity of the proposed scheme to the highly distorted signal is demonstrated through the experimental results shown in Fig. 11. As in Fig.7(a), the three-phase grid voltage signal shown in Fig. 11(a) is characterized by presence of $5^{\text {th }}, 7^{\text {th }}, 11^{\text {th }}$ and $13^{\text {th }}$ order harmonic components with amplitudes of $30 \%, 10 \%, 8 \%$ and $7 \%$, respectively, with respect to the fundamental. Also, a frequency jump of $50 \mathrm{~Hz}$ to $45 \mathrm{~Hz}$ is introduced similar to that shown in Fig. 7(a). Both these variation in supply voltages are considered along with the presence of dc offset $10 \%$. Fig. 11(b) shows that the

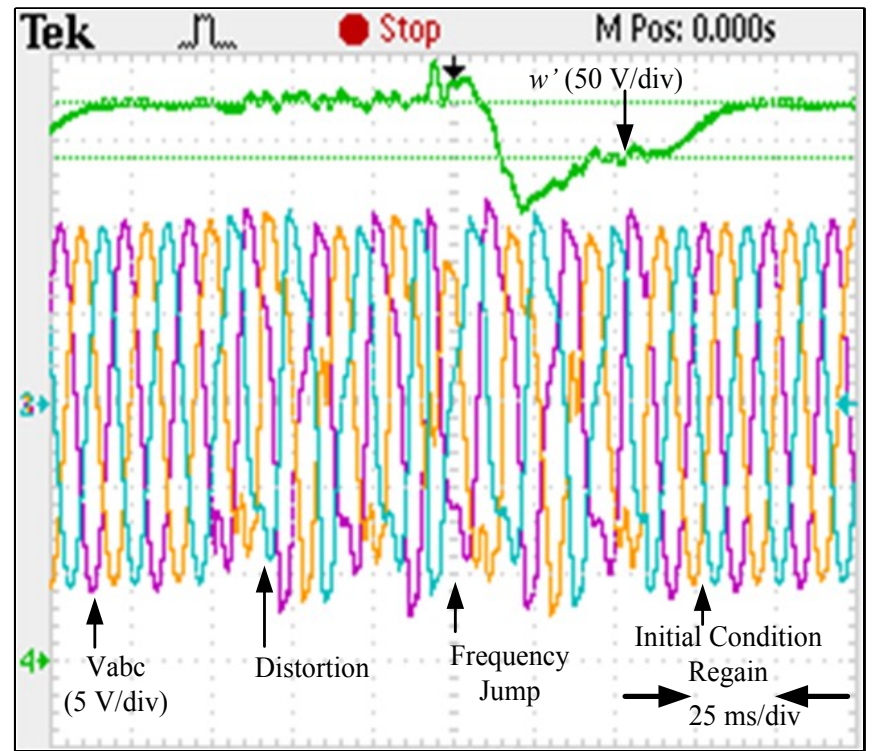

(a)

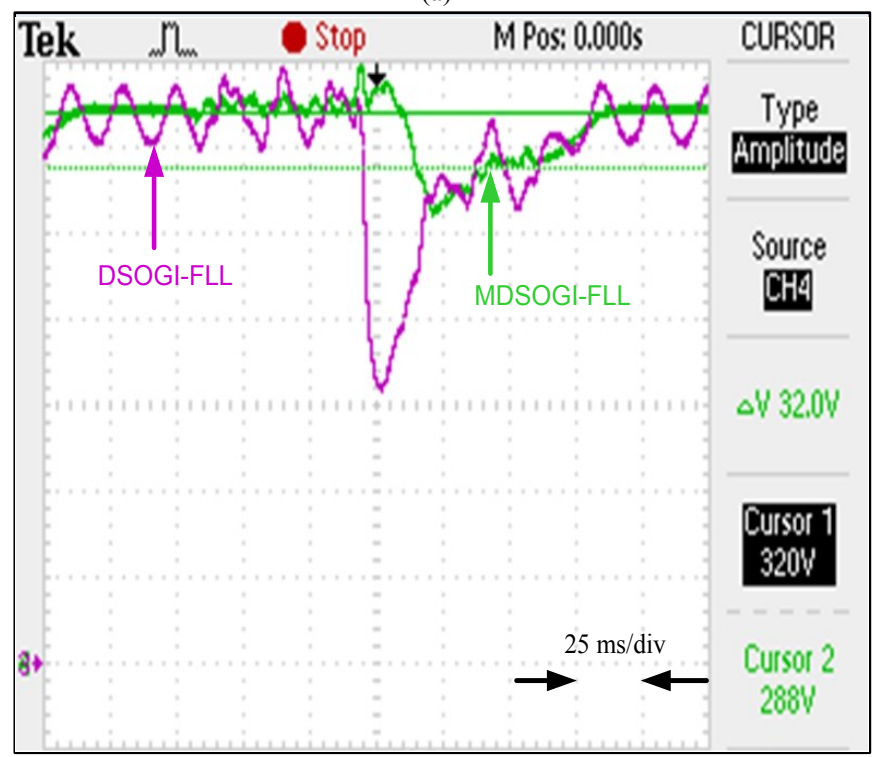

(b)

Fig. 11. Experimental results with dc offset and harmonics in supply voltage: (a) Three-phase supply voltage (b) Estimated frequency with DSOGI-FLL and MDSOGI-FLL.

estimated frequency of DSOGI-FLL not only has the $100 \mathrm{~Hz}$ frequency component, but also shows the harmonic distortion due to the effect of harmonics present in the supply voltage. Unlike it, the estimated frequency of MDSOGI-FLL is once again free from the effect of dc offset and exhibits a superior performance with a very little deviation from the average value. The negligible high frequency ripple is due to the harmonics in the supply voltage.

The proposed technique works equally well even in case when the input voltage is characterized by multiple abnormalities. To illustrate the performance of the MDSOGIFLL under such multiple abnormalities, a supply voltage having harmonics, voltage sag and dc-offset all together, is considered. Fig. 12(a) shows the input voltage signal, which is characterized by the presence of the $5^{\text {th }}, 7^{\text {th }}, 11^{\text {th }}$ and $13^{\text {th }}$ order harmonic components with the amplitudes of $30 \%, 10 \%, 8 \%$ 


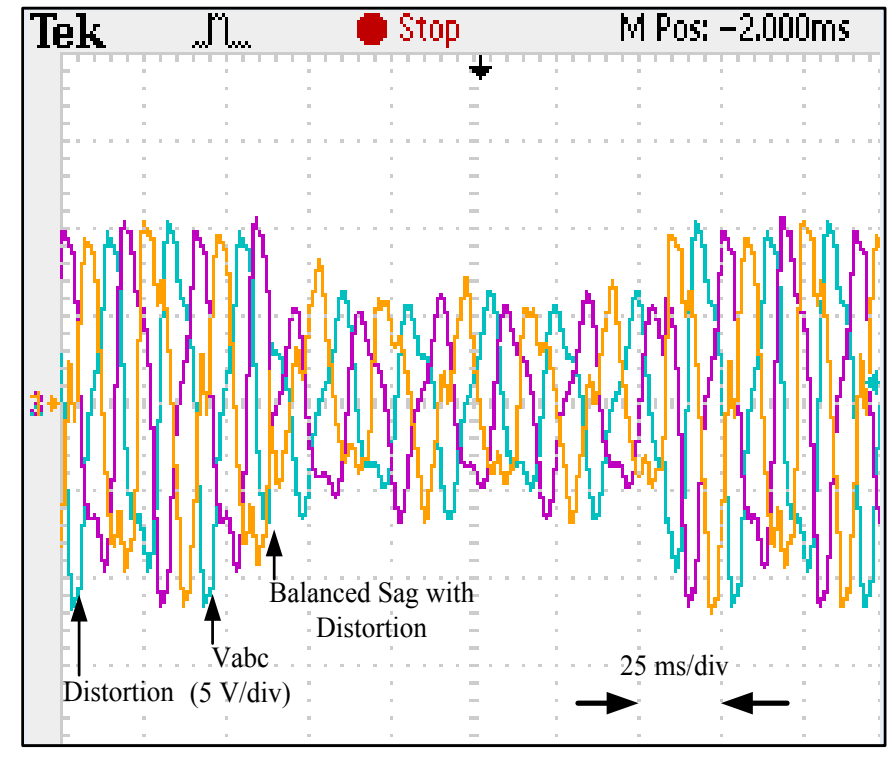

(a)

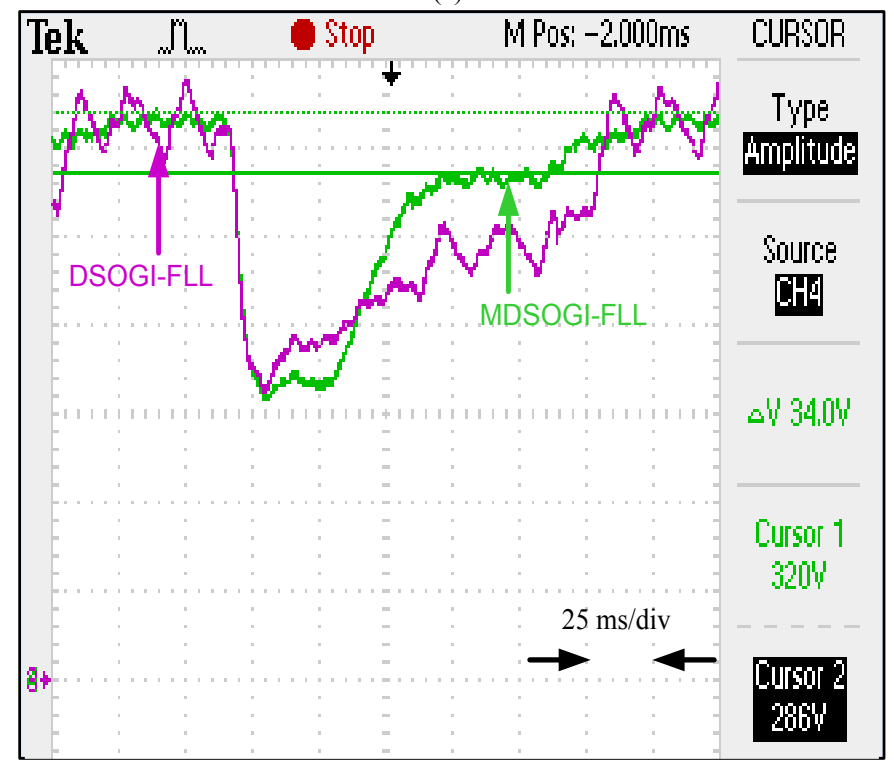

(b)

Fig. 12. Experimental results with input voltage characterized simultaneously by dc offset, harmonics and voltage sag: (a) Three-phase supply voltage (b) Estimated frequency with DSOGI-FLL and MDSOGI-FLL.

and $7 \%$ (with respect to fundamental component), respectively along-with the $10 \%$ of dc offset error. Further a step change (decrease/sag) of $50 \%$ is applied in magnitude of supply voltage. Fig. 12 (b) highlights the performance of the proposed MDSOGI-FLL in estimating the grid voltage signal frequency and once again MDSOGI-FLL is found to be superior over DSOGI-FLL. With MDSOGI-FLL, the estimated frequency is not only estimated quickly but is also free from oscillatory nature, unlike that with the DSOGI-FLL technique.

\section{CONCLUSION}

DSOGI-FLL can accurately estimate the frequency of the grid signal under various abnormal conditions except the presence of dc offset. The presence of dc offset in grid voltage introduces a ripple of $100 \mathrm{~Hz}$ low frequency component in the estimated frequency. This $100 \mathrm{~Hz}$ ripple is further having the distortion when harmonics are present. This error in the estimation of frequency may affect the synchronization and control of DG based inverter.

The proposed MDSOGI-FLL structure, which consists of the three fundamental blocks: 1) basic SOGI-building block with third integrator; 2) FLL block to estimate the frequency adaptively and 3) PSC block to calculate the positive sequence components; eliminates the ripple present in the estimated synchronized frequency. In MDSOGI-FLL, the third integrator added to the standard SOGI-QSG structure attenuates the dc offset. As a result, in addition to the benefits of the conventional DSOGI-FLL, the proposed method exhibits the capability of rejecting dc offset and hence, can accurately track the frequency of fundamental component of grid-voltage effectively under all grid abnormalities. Simulation and experimental results justify that the proposed MDOSGI-FLL is accurate and shows better transient response than that of DSOGI-FLL.

\section{REFERENCES}

[1] F. Blaabjerg, Zhe Chen, and S. Baekhoej Kjaer, "Power electronics as efficient interface in dispersed power generation systems, "IEEE Trans. on Power Electron., vol. 19, no. 5, pp. 1184-1194, September 2004.

[2] F. Blaabjerg, Remus Teodorescu, Marco Liserre, and Adrian V. Timbus, "Overview of control and grid synchronization for distributed power generation systems", IEEE Trans. on Ind. Electron., vol. 53, no. 5, pp. 1398-1409, October 2006.

[3] Se-Kyo Chung, "A phase tracking system for three phase utility interface inverters," IEEE Trans. Power Electron., vol. 15, no. 3, pp. 431-438, May 2000.

[4] Benjamin Kroposki, Christopher Pink, Richard DeBlasio, Marcelo Simoes, Holly Thomas and Pankaj K. Sen "Benefits of power electronic interfaces for distributed energy systems," IEEE Trans. on Energy Conv., vol. 25, no. 3, pp. September 2010.

[5] Maohai Wang and Yuanzhang Sun, "A practical method to improve phasor and power measurement accuracy of DFT algorithm," in IEEE Trans. on Power Del., vol. 21, no. 3, pp. 1054-1062, July 2006.

[6] H. A. Darwish and M. Fikri, "Practical considerations for recursive DFT implementation in numerical relays," in IEEE Trans. on Power Del., vol. 22, no. 1, pp. 42-49, Jan 2007.

[7] H. Wen, Z. Teng, Y. Wang and X. Hu, "Spectral correction approach based on desirable sidelobe window for harmonic analysis of industrial power system," in IEEE Trans. on Ind. Electron., vol. 60, no. 3, pp. 1001-1010, March 2013.

[8] R. Teodorescu, M. Liserre and P. Rodriguez, "A thesis on grid converters for photovoltaic and wind power systems," 2011, ISBN: 9780-470-05751-3

[9] Md. Shamim Reza, Mihai Ciobotaru and V. G. Agelidis, "Accurate estimation of single-phase grid voltage parameters under distorted conditions," IEEE Trans. Power Del. vol. 29, no. 3, pp. 1138-1146, June-2014.

[10] Fengjiang Wu, Lujie Zhang, and Jiandong Duan, "A new two-phase stationary-frame-based enhanced PLL for three-phase grid synchronization," IEEE Trans. Circuits Syst. II, Express Briefs, vol. 62, no. 3, March 2015.

[11] Y. Han, M. Luo, X. Zhao, J. M. Guerrero and L. Xu, "Comparative performance evaluation of orthogonal-signal-generators-based singlephase PLL algorithms-A survey," in IEEE Trans. on Power Electron., vol. 31, no. 5, pp. 3932-3944, May 2016.

[12] V. Kaura and V. Blasco, "Operation of a phase locked loop system under distorted utility conditions," IEEE Trans. Ind. Appl., vol. 33, no.1, pp. 58-63, Jan 1997.

[13] P. Rodriguez, J. Pou, J. Bergas, J. I. Candela, R. P. Burgos and D. Boroyevich, "Decoupled double synchronous reference frame PLL for power converters control," in IEEE Trans. on Power Electron., vol. 22, no. 3, pp. 1078-1078, May 2007. 
[14] P. Rodriguez, A. Luna, I. Candela, R. Teodorescu, and F. Blaabjerg, "Grid synchronization of power converters using multiple second-order generalized integrators," in Proc. $34^{\text {th }}$ Annu. Conf. IEEE Ind. Electron., pp. 755-760, November 2008.

[15] P. Rodriguez, A. Luna, R. Santiago M. Aguilar, I. E. Otadui, R. Teodorescu, and F. Blaabjerg, "A stationary reference frame grid synchronization system for three-phase grid-connected power converters under adverse grid conditions," IEEE Trans. Power Electron., vol. 27, no. 1, pp. 99-112, January 2012.

[16] Saeed Golestan, Malek Ramezani, Josep. M. Guerrer, and Mohammad Monfared, " $d q$-Frame Cascaded Delayed Signal Cancellation- Based PLL: Analysis, Design, and Comparison With Moving Average FilterBased PLL," IEEE Trans. Power Electron., vol. 30, no. 3, pp. 16181631, March 2015

[17] Md. Shamim Reza, Mihai Ciobotaru, and V. G. Agelidis, "Power system frequency estimation by using a Newton-type technique for smart meters," IEEE Trans. Instrum. Meas., vol. 64, no. 3, pp. 615-624, March -2015.

[18] J. Matas, M. Castilla, J. Miret, L. Garcia de Vicuna, and R. Guzman, "An adaptive prefiltering method to improve the speed/accuracy tradeoff of voltage sequence detection methods under adverse grid conditions," IEEE Trans. on Ind. Electron., vol. 61, no. 5, pp. 2139-2151, May-2014.

[19] M. Ciobotaru, R. Teodorescu and V. G. Agelidis, "Offset rejection for PLL based synchronization in grid-connected converters," Proc. $23^{\text {rd }}$ Annu. IEEE Appl. Power Energy Conf. Expo., pp. 1611-1617, February2008.

[20] M. Karimi Ghartemani, S. Khajehoddin, P. Jain, and A. Bakhshai. "Comparison of two methods for addressing dc component in phaselocked loop (PLL) systems," in Proc. IEEE ECCE, pp. 3053-3058, September 2011.

[21] M. Karimi-Ghartemani, S. Khajehoddin, P. Jain, A. Bakhshai and M. Mojiri, "Addressing dc component in PLL and notch filter algorithms," IEEE Trans. Power Electron., vol. 27, no. 1, pp. 78-86 January 2012.

[22] S. Hwang, L. Liu, H. Li, and J. M. Kim, "DC offset error compensation for synchronous reference frame PLL in single-phase grid-connected converters," IEEE Trans. Power Electron., vol. 27, no. 8, pp. 34673471, August 2012.

[23] Fengjiang Wu, Dongyang Sun, Lujie Zhang and Jiandong Duan, "Influence of plugging DC offset estimation integrator in single-phase EPLL and alternative scheme to eliminate effect of input DC offset and harmonics," IEEE Trans. on Ind. Electron., vol. 62, no. 8, pp. 48234831, May-2014.

[24] S. Golestan, Josep M. Guerrero and G.B. Gharehpetian, "Five approaches to deal with problem of DC offset in phase-locked loop algorithm: Design consideration and performance evaluation," IEEE Trans. Power Electron. , vol. 31, no. 1, pp. 648-661, January 2016.

[25] K. R. Patil and Hiren H. Patel, "Modified dual second-order generalised integrator FLL for synchronization of a distributed generator to a weak grid," in Proc. $16^{\text {th }}$ Annu. Conf. IEEE-EEEIC, June 2016.

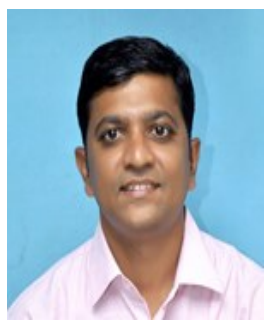

Kalpeshkumar Patil received the B.E. degree in electrical engineering from the Shri S.V. Mandal Institute of Technology, South Gujarat University, Surat, India, in 2003, and the M.Tech. degree in electrical engineering systems in 2009 from the S.V. National Institute of Technology, Surat, India.

He is currently working as an Assistant Professor in Department of Electrical Engineering at Sarvajanik College of Engineering \& Technology, Surat and pursuing the part time Ph.D. degree course in electrical engineering affiliated to Gujarat Technological University, Ahmedabad, Gujarat, India. His current research interests include grid synchronization, active power filtering, power quality issues.

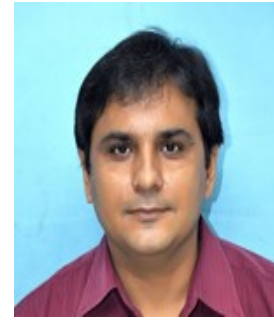

Hiren Patel received the B.E. degree in electrical engineering from the S.V. Regional College of Engineering and Technology (now S.V. National Institute of Technology), South Gujarat University, Surat, India, in 1996, and the M.Tech. degree in energy systems in 2003 from the Indian Institute of Technology_Bombay (IITB),Mumbai, India. He has received his $\mathrm{Ph}$. D. degree from the Indian Institute of Technology. He is working as a Professor at Sarvajanik College of Engineering and Technology, Surat.

His current research interests include computer aided simulation techniques, distributed generation, and renewable energy, especially energy extraction from photovoltaic arrays. Mr. Patel is a Life Member of the Indian Society for Technical Education. 